\title{
Persistence of the spectral gap for the Landau-Pekar equations
}

\author{
Dario Feliciangeli $^{1}$ (D) $\cdot$ Simone Rademacher ${ }^{1} \cdot$ Robert Seiringer $^{1}$
}

Received: 5 October 2020 / Revised: 2 December 2020 / Accepted: 22 December 2020 /

Published online: 11 February 2021

(c) The Author(s) 2021

\begin{abstract}
The Landau-Pekar equations describe the dynamics of a strongly coupled polaron. Here, we provide a class of initial data for which the associated effective Hamiltonian has a uniform spectral gap for all times. For such initial data, this allows us to extend the results on the adiabatic theorem for the Landau-Pekar equations and their derivation from the Fröhlich model obtained in previous works to larger times.
\end{abstract}

Keywords Polaron · Dynamics · Schrödinger operator · Quantized field

Mathematics Subject Classification 35Q41 · 35Q40 · 46N50

\section{Introduction and main results}

The Landau-Pekar equations [5] provide an effective description of the dynamics for a strongly coupled polaron, modeling an electron moving in an ionic crystal. The strength of the interaction of the electron with its self-induced polarization field is described by a coupling parameter $\alpha>0$. In this system of coupled differential equations, the time evolution of the electron wave function $\psi_{t} \in H^{1}\left(\mathbb{R}^{3}\right)$ is governed by a Schrödinger equation with respect to an effective Hamiltonian $h_{\varphi_{t}}$ depending on the polarization field $\varphi_{t} \in L^{2}\left(\mathbb{R}^{3}\right)$, which evolves according to a classical field equation. Motivated by the recent work in $[7,8,10]$, we are interested in initial data for which the Hamiltonian

Dario Feliciangeli

dario.feliciangeli@ist.ac.at

Simone Rademacher

simone.rademacher@ist.ac.at

Robert Seiringer

robert.seiringer@ist.ac.at

1 Institute of Science and Technology Austria (IST Austria), Am Campus 1, 3400 Klosterneuburg, Austria 
$h_{\varphi_{t}}$ possesses a uniform spectral gap (independent of $t$ and $\alpha$ ) above the infimum of its spectrum.

The Landau-Pekar equations are of the form

$$
\begin{aligned}
i \partial_{t} \psi_{t} & =h_{\varphi_{t}} \psi_{t} \\
i \alpha^{2} \partial_{t} \varphi_{t} & =\varphi_{t}+\sigma_{\psi_{t}}
\end{aligned}
$$

with

$$
\begin{aligned}
& h_{\varphi}=-\Delta+V_{\varphi}, \quad V_{\varphi}(x)=2(2 \pi)^{3 / 2} \operatorname{Re}\left[(-\Delta)^{-1 / 2} \varphi\right](x), \\
& \sigma_{\psi}(x)=(2 \pi)^{3 / 2}\left[(-\Delta)^{-1 / 2}|\psi|^{2}\right](x) .
\end{aligned}
$$

For initial data $\left(\psi_{0}, \varphi_{0}\right) \in H^{1}\left(\mathbb{R}^{3}\right) \times L^{2}\left(\mathbb{R}^{3}\right),(1)$ is well-posed for all times $t \in \mathbb{R}$ (see [1] or Lemma 1 below).

For $(\psi, \varphi) \in H^{1}\left(\mathbb{R}^{3}\right) \times L^{2}\left(\mathbb{R}^{3}\right)$ with $\|\psi\|_{2}=1$, the energy functional corresponding to the Landau-Pekar equations is defined as

$$
\mathcal{G}(\psi, \varphi)=\left\langle\psi, h_{\varphi} \psi\right\rangle+\|\varphi\|_{2}^{2} .
$$

One readily checks that for solutions of (1), $\mathcal{G}\left(\psi_{t}, \varphi_{t}\right)$ is independent of $t$ [1, Lemma 2.1], and the same holds for $\left\|\psi_{t}\right\|_{2}$. We also define

$$
\mathcal{E}(\psi)=\inf _{\varphi \in L^{2}\left(\mathbb{R}^{3}\right)} \mathcal{G}(\psi, \varphi), \quad \mathcal{F}(\varphi)=\inf _{\substack{\psi \in H^{1}\left(\mathbb{R}^{3}\right) \\\|\psi\|_{2}=1}} \mathcal{G}(\psi, \varphi)
$$

These three functionals are known as Pekar functionals and we shall discuss some of their properties in Sect. 2. It follows from the work in [9] that there exist $\left(\psi_{\mathrm{P}}, \varphi_{\mathrm{P}}\right) \in$ $H^{1}\left(\mathbb{R}^{3}\right) \times L^{2}\left(\mathbb{R}^{3}\right)$ with $\left\|\psi_{\mathrm{P}}\right\|_{2}=1$, called Pekar minimizers, realizing

$$
\inf _{\psi, \varphi} \mathcal{G}(\psi, \varphi)=\mathcal{G}\left(\psi_{\mathrm{P}}, \varphi_{\mathrm{P}}\right)=\mathcal{E}\left(\psi_{\mathrm{P}}\right)=\mathcal{F}\left(\varphi_{\mathrm{P}}\right)=e_{\mathrm{P}}<0
$$

and $\left(\psi_{\mathrm{P}}, \varphi_{\mathrm{P}}\right)$ is unique up to symmetries (i.e., translations and multiplication of $\psi_{\mathrm{P}}$ by a constant phase factor). We also note that the Hamiltonian $h_{\varphi_{\mathrm{P}}}$ has a spectral gap above its ground state energy, i.e., $\Lambda\left(\varphi_{\mathrm{P}}\right)>0$, where we denote for general $\varphi \in L^{2}\left(\mathbb{R}^{3}\right)$

$$
\Lambda(\varphi)=\inf _{\substack{\lambda \in \operatorname{spec}\left(h_{\varphi}\right) \\ \lambda \neq e(\varphi)}}|\lambda-e(\varphi)| \text { with } e(\varphi)=\inf \operatorname{spec} h_{\varphi}
$$

In the following we consider solutions $\left(\psi_{t}, \varphi_{t}\right)$ to the Landau-Pekar equations (1) with initial data $\left(\psi_{0}, \varphi_{0}\right)$ such that its energy $\mathcal{G}\left(\psi_{0}, \varphi_{0}\right)$ is sufficiently close to $e_{\mathrm{P}}$, and show that for such initial data the Hamiltonian $h_{\varphi_{t}}$ possesses a uniform spectral gap above the infimum of its spectrum for all times $t \in \mathbb{R}$ and any coupling constant $\alpha>0$. This is the content of the following Theorem. 
Theorem 1 For any $0<\Lambda<\Lambda\left(\varphi_{\mathrm{P}}\right)$ there exists $\varepsilon_{\Lambda}>0$ such that if $\left(\psi_{t}, \varphi_{t}\right)$ is the solution of the Landau-Pekar equations (1) with initial data $\left(\psi_{0}, \varphi_{0}\right) \in H^{1}\left(\mathbb{R}^{3}\right) \times$ $L^{2}\left(\mathbb{R}^{3}\right)$ with $\left\|\psi_{0}\right\|_{2}=1$ and $\mathcal{G}\left(\psi_{0}, \varphi_{0}\right) \leq e_{\mathrm{P}}+\varepsilon_{\Lambda}$, then

$$
\Lambda\left(\varphi_{t}\right) \geq \Lambda \text { for all } t \in \mathbb{R}, \alpha>0 \text {. }
$$

Theorem 1 is proved in Sect. 3. It provides a class of initial data for the LandauPekar equations for which the Hamiltonian $h_{\varphi_{t}}$ has a uniform spectral gap for all times $t \in \mathbb{R}$. The existence of initial data with this particular property is of relevance for recent work $[7,8,10]$ on the adiabatic theorem for the Landau-Pekar equations, and on their derivation from the Fröhlich model (where the polarization is described as a quantum field instead). For this particular initial data, the results obtained there can then be extended in the following way:

Adiabatic theorem. Due to the separation of time scales in (1), the Landau-Pekar equations decouple adiabatically for large $\alpha$ (see [8] or also [2] for an analogous onedimensional model). To be more precise, in [8] the initial phonon state function is assumed to satisfy

$$
\varphi_{0} \in L^{2}\left(\mathbb{R}^{3}\right) \text { with } e\left(\varphi_{0}\right)=\inf \operatorname{spec} h_{\varphi_{0}}<0,
$$

which implies that $h_{\varphi_{0}}$ has a spectral gap and that there exists a unique positive and normalized ground state $\psi_{\varphi_{0}}$ of $h_{\varphi_{0}}$. Under this assumption, denoting by $\left(\psi_{t}, \varphi_{t}\right)$ the solution of the Landau-Pekar equations (1) with initial data $\left(\psi_{\varphi_{0}}, \varphi_{0}\right)$, [8, Thm. II.1 $\&$ Rem. II.3] proves that there exist constants $C, T>0$ (depending on $\varphi_{0}$ ) such that

$$
\left\|\psi_{t}-e^{-i \int_{0}^{t} d s e\left(\varphi_{s}\right)} \psi_{\varphi_{t}}\right\|_{2}^{2} \leq C \alpha^{-4} \text { for all }|t| \leq T \alpha^{2}
$$

where $\psi_{\varphi_{t}}$ denotes the unique positive and normalized ground state of $h_{\varphi_{t}}$. The restriction on $|t|$ in (9) is due to the need of ensuring that the spectral gap of the effective Hamiltonian $h_{\varphi_{t}}$ does not become too small for initial data satisfying (8), which is only proven (in [8, Lemma II.1]) for times $|t| \leq T \alpha^{2}$. Nevertheless, assuming that there exists $\Lambda>0$ such that $\Lambda\left(\varphi_{t}\right)>\Lambda$ for all times $t \in \mathbb{R}$, the adiabatic theorem in [8, Thm. II.1] allows to approximate $\psi_{t}$ by $e^{-i \int_{0}^{t} d s e\left(\varphi_{s}\right)} \psi_{\varphi_{t}}$ for all times $|t| \ll \alpha^{4}$. This raises the question about initial data for which the existence of a spectral gap of order one holds true for longer times, and Theorem 1 answers this question. In fact, by suitably adjusting the phase factor, we can prove the following stronger result.

Corollary 1 Let $\varphi_{0} \in L^{2}\left(\mathbb{R}^{3}\right)$ be such that

$$
\mathcal{F}\left(\varphi_{0}\right) \leq e_{\mathrm{P}}+\varepsilon
$$

for sufficiently small $\varepsilon>0$. Then, $h_{\varphi_{0}}$ has a ground state $\psi_{\varphi_{0}}$. Let $\left(\psi_{t}, \varphi_{t}\right)$ be the solution to the Landau-Pekar equations (1) with initial data $\left(\psi_{\varphi_{0}}, \varphi_{0}\right)$ and define

$$
v(s)=-\alpha^{-4}\left\langle\psi_{\varphi_{s}}, V_{\operatorname{Im} \varphi_{s}} R_{\varphi_{s}}^{3} V_{\operatorname{Im} \varphi_{s}} \psi_{\varphi_{s}}\right\rangle \text { and } \widetilde{\psi}_{t}=e^{i \int_{0}^{t} d s\left(e\left(\varphi_{s}\right)+v(s)\right)} \psi_{t},
$$


where $R_{\varphi_{s}}=q_{s}\left(h_{\varphi_{s}}-e\left(\varphi_{s}\right)\right)^{-1} q_{s}$ with $q_{s}=1-\left|\psi_{\varphi_{s}}\right\rangle\left\langle\psi_{\varphi_{s}}\right|$. Then, there exists $a$ $C>0$ (independent of $\varphi_{0}$ and $\alpha$ ) such that

$$
\left\|\widetilde{\psi}_{t}-\psi_{\varphi_{t}}\right\|_{2}^{2} \leq C \varepsilon \alpha^{-4}\left(1+\alpha^{-2}|t|\right) e^{C \alpha^{-4}|t|} .
$$

Our proof in Sect. 3 shows that the smallness condition on $\varepsilon$ in Corollary 1 can be made explicit in terms of properties of $\varphi_{\mathrm{P}}$. It also shows that $\min _{\theta \in[0,2 \pi)} \| e^{i \theta} \psi_{t}-$ $\psi_{\varphi_{t}} \|_{2}^{2} \leq C \varepsilon$ for all times $t$, independently of $\alpha$. The bound (12) improves upon this for large $\alpha$ as long as $\alpha^{-4}|t| e^{C \alpha^{-4}|t|} \ll \alpha^{2}$ and hence, in particular, for $|t| \lesssim \alpha^{4}$.

Effective dynamics for the Fröhlich Hamiltonian. As already mentioned, the LandauPekar equations provide an effective description of the dynamics for a strongly coupled polaron. Its true dynamics is described by the Fröhlich Hamiltonian [4] $H_{\alpha}$ acting on $L^{2}\left(\mathbb{R}^{3}\right) \otimes \mathcal{F}$, the tensor product of the Hilbert space $L^{2}\left(\mathbb{R}^{3}\right)$ for the electron and the bosonic Fock space $\mathcal{F}$ for the phonons. We refer to [7,8] for a detailed definition. Pekar product states of the form $\psi_{t} \otimes W\left(\alpha^{2} \varphi_{t}\right) \Omega$, with $\left(\psi_{t}, \varphi_{t}\right)$ a solution of the LandauPekar equations, $W$ the Weyl operator and $\Omega$ the Fock space vacuum, were proven in [8, Thm. II.2] to approximate the dynamics defined by the Fröhlich Hamiltonian $H_{\alpha}$ for times $|t| \ll \alpha^{2}$. Recently, it was shown in [7] that in order to obtain a norm approximation valid for times of order $\alpha^{2}$, one needs to implement correlations among phonons, which are captured by a suitable Bogoliubov dynamics acting on the Fock space of the phonons only. In fact, considering initial data satisfying (8), [7, Theorem I.3] proves that there exist constants $C, T>0$ (depending on $\varphi_{0}$ ) such that

$$
\begin{aligned}
& \left\|e^{-i H_{\alpha} t} \psi_{\varphi_{0}} \otimes W\left(\alpha^{2} \varphi_{0}\right) \Omega-e^{-i \int_{0}^{t} d s \omega(s)} \psi_{t} \otimes W\left(\alpha^{2} \varphi_{t}\right) \Upsilon_{t}\right\|_{L^{2}\left(\mathbb{R}^{3}\right) \otimes \mathcal{F}} \leq C \alpha^{-1} \\
& \quad \text { for all }|t| \leq T \alpha^{2}
\end{aligned}
$$

where $\omega(s)=\alpha^{2} \operatorname{Im}\left\langle\varphi_{s}, \partial_{s} \varphi_{s}\right\rangle+\left\|\varphi_{s}\right\|_{2}^{2}$ and $\Upsilon_{t}$ is the solution of the dynamics of a suitable Bogoliubov Hamiltonian on $\mathcal{F}$ (see [7, Definition I.2] for a precise definition). As for the adiabatic theorem discussed above, the restriction to times $|t| \leq T \alpha^{2}$ results from the need of a spectral gap of $h_{\varphi_{t}}$ of order one (compare with [7, Remark I.4]), which under the sole assumption (8) is guaranteed by [8, Lemma II.1] only for $|t| \leq T \alpha^{2}$. Theorem 1 now provides a class of initial data for which the above norm approximation holds true for all times of order $\alpha^{2}$, in the following sense.

Corollary 2 Let $\varphi_{0} \in L^{2}\left(\mathbb{R}^{3}\right)$ be such that

$$
\mathcal{F}\left(\varphi_{0}\right) \leq e_{\mathrm{P}}+\varepsilon
$$

for sufficiently small $\varepsilon>0$. Then, $h_{\varphi_{0}}$ has a ground state $\psi_{\varphi_{0}}$. Let $\left(\psi_{t}, \varphi_{t}\right)$ be the solution to the Landau-Pekar equations (1) with initial data $\left(\psi_{\varphi_{0}}, \varphi_{0}\right)$. Then, there exists a $C>0$ (independent of $\varphi_{0}$ and $\alpha$ ) such that

$$
\left\|e^{-i H_{\alpha} t} \psi_{\varphi_{0}} \otimes W\left(\alpha^{2} \varphi_{0}\right) \Omega-e^{-i \int_{0}^{t} d s \omega(s)} \psi_{t} \otimes W\left(\alpha^{2} \varphi_{t}\right) \Upsilon_{t}\right\|_{L^{2}\left(\mathbb{R}^{3}\right) \otimes \mathcal{F}} \leq C \alpha^{-1} e^{C \alpha^{-2}|t|} .
$$


Again, the smallness condition on $\varepsilon$ in Corollary 2 can be made explicit in terms of properties of $\varphi_{\mathrm{P}}$. Corollary 2 is an immediate consequence of Theorem 1 and the method of proof in [7], as explained in [7, Remark I.4].

\section{Properties of the spectral gap and the Pekar functionals}

Throughout the paper, we use the symbol $C$ for generic constants, and their value might change from one occurrence to the next.

\subsection{Preliminary Lemmas}

We begin by stating some preliminary Lemmas we shall need throughout the following discussion.

Lemma 1 (Lemma 2.1 in [1]) For any $\left(\psi_{0}, \varphi_{0}\right) \in H^{1}\left(\mathbb{R}^{3}\right) \times L^{2}\left(\mathbb{R}^{3}\right)$, there is a unique global solution $\left(\psi_{t}, \varphi_{t}\right)$ of the Landau-Pekar equations (1). Moreover, $\left\|\psi_{0}\right\|_{2}=\left\|\psi_{t}\right\|_{2}, \mathcal{G}\left(\psi_{0}, \varphi_{0}\right)=\mathcal{G}\left(\psi_{t}, \varphi_{t}\right)$ for all $t \in \mathbb{R}$ and there exists a constant $C>0$ such that

$$
\left\|\psi_{t}\right\|_{H^{1}\left(\mathbb{R}^{3}\right)} \leq C, \quad\left\|\varphi_{t}\right\|_{2} \leq C
$$

for all $\alpha>0$ and all $t \in \mathbb{R}$.

The following Lemma collects some properties of $V_{\varphi}$ and $\sigma_{\psi}$ (see also [8, Lemma III.2] and [7, Lemma II.2]).

Lemma 2 There exists $C>0$ such that for every $\varphi \in L^{2}\left(\mathbb{R}^{3}\right)$ and $\psi \in H^{1}\left(\mathbb{R}^{3}\right)$

$$
\left\|V_{\varphi}\right\|_{6} \leq C\|\varphi\|_{2}, \quad\left\|V_{\varphi} \psi\right\|_{2} \leq C\|\varphi\|_{2}\|\psi\|_{H^{1}\left(\mathbb{R}^{3}\right)}
$$

and with the additional assumption $\|\psi\|_{2}=1$

$$
\|\psi\|_{H^{1}\left(\mathbb{R}^{3}\right)}^{2} \leq 2\left\langle\psi, h_{\varphi} \psi\right\rangle+C\left(\|\varphi\|_{2}^{2}+1\right)
$$

Moreover, there exists $C>0$ such that for all $\psi_{1}, \psi_{2} \in H^{1}\left(\mathbb{R}^{3}\right)$

$$
\left\|\sigma_{\psi_{1}}-\sigma_{\psi_{2}}\right\|_{2} \leq C\left(\left\|\psi_{1}\right\|_{2}+\left\|\psi_{2}\right\|_{2}\right) \min _{\theta \in[0,2 \pi)}\left\|e^{i \theta} \psi_{1}-\psi_{2}\right\|_{H^{1}\left(\mathbb{R}^{3}\right)} .
$$

Proof The first two inequalities in (17) follow immediately from [8, Lemma III.2] and [7, Lemma II.2]. In order to prove (18), let $\varepsilon>0$, then

$$
\begin{aligned}
\|\psi\|_{H^{1}\left(\mathbb{R}^{3}\right)}^{2} & =\left\langle\psi, h_{\varphi_{0}} \psi\right\rangle-\left\langle\psi, V_{\varphi_{0}} \psi\right\rangle+1 \\
& \leq\left\langle\psi, h_{\varphi_{0}} \psi\right\rangle+\varepsilon\|\psi\|_{H^{1}\left(\mathbb{R}^{3}\right)}^{2}+C \varepsilon^{-1}\left\|\varphi_{0}\right\|_{2}^{2}+1 .
\end{aligned}
$$

Hence, choosing $\varepsilon=1 / 2$, we arrive at (18). 
For (19), we note that $\sigma_{\psi}=\sigma_{e^{i \theta} \psi}$ for arbitrary $\theta \in \mathbb{R}$. Hence, it is enough to prove the result for $\theta=0$. We write the difference

$$
\begin{aligned}
\widehat{\sigma}_{\psi_{1}}(k)-\widehat{\sigma}_{\psi_{2}}(k) & =|k|^{-1}\left(\left\langle\psi_{1}, e^{-i k \cdot} \psi_{1}\right\rangle-\left\langle\psi_{2}, e^{-i k \cdot} \psi_{2}\right\rangle\right) \\
& =|k|^{-1}\left(\left\langle\psi_{1}-\psi_{2}, e^{-i k \cdot} \psi_{1}\right\rangle+\left\langle\psi_{2}, e^{-i k \cdot}\left(\psi_{1}-\psi_{2}\right)\right\rangle\right),
\end{aligned}
$$

where $\widehat{\sigma}_{\psi}(k)=(2 \pi)^{-3 / 2} \int d x e^{-i k \cdot x} \sigma_{\psi}(x)$ denotes the Fourier transform of $\sigma_{\psi}$. Thus,

$$
\left\|\sigma_{\psi_{1}}-\sigma_{\psi_{2}}\right\|_{2}^{2} \leq 2 \int d k \frac{1}{|k|^{2}}\left(\left|\left\langle\psi_{1}-\psi_{2}, e^{-i k \cdot} \psi_{1}\right\rangle\right|^{2}+\left|\left\langle\psi_{2}, e^{-i k \cdot}\left(\psi_{1}-\psi_{2}\right)\right\rangle\right|^{2}\right) .
$$

For the first term, we write

$$
\begin{aligned}
& \int \frac{d k}{|k|^{2}}\left|\left\langle\psi_{1}-\psi_{2}, e^{-i k \cdot} \psi_{1}\right\rangle\right|^{2} \\
& \quad=C \int \frac{d x d y}{|x-y|}\left(\psi_{1}-\psi_{2}\right)(x) \overline{\left(\psi_{1}-\psi_{2}\right)(y)} \overline{\psi_{1}(x)} \psi_{1}(y) .
\end{aligned}
$$

The Hardy-Littlewood-Sobolev inequality implies that

$$
\begin{aligned}
& \int \frac{d k}{|k|^{2}}\left|\left\langle\psi_{1}-\psi_{2}, e^{-i k \cdot} \psi_{1}\right\rangle\right|^{2} \\
& \quad \leq C\left\|\psi_{1} \overline{\left(\psi_{1}-\psi_{2}\right)}\right\|_{6 / 5}^{2} \leq C\left\|\psi_{1}-\psi_{2}\right\|_{3}^{2}\left\|\psi_{1}\right\|_{2}^{2},
\end{aligned}
$$

and we obtain with the Sobolev inequality that

$$
\int \frac{d k}{|k|^{2}}\left|\left\langle\psi_{1}-\psi_{2}, e^{-i k \cdot} \psi_{1}\right\rangle\right|^{2} \leq C\left\|\psi_{1}-\psi_{2}\right\|_{H^{1}\left(\mathbb{R}^{3}\right)}^{2}\left\|\psi_{1}\right\|_{2}^{2}
$$

The second term of (22) can be bounded in a similar way, and we obtain the desired estimate.

We recall the definition of the reduced resolvent

$$
R_{\varphi}=q_{\psi_{\varphi}}\left(h_{\varphi}-e(\varphi)\right)^{-1} q_{\psi_{\varphi}}
$$

where $q_{\psi_{\varphi}}=1-\left|\psi_{\varphi}\right\rangle\left\langle\psi_{\varphi}\right|$. In the following Lemma we collect useful estimates on $R_{\varphi}$.

Lemma 3 There exists $C>0$ such that

$$
\left\|R_{\varphi}\right\|=\Lambda(\varphi)^{-1}, \quad\left\|(-\Delta+1)^{1 / 2} R_{\varphi}^{1 / 2}\right\| \leq C\left(1+\|\varphi\|_{2}\left\|R_{\varphi}^{1 / 2}\right\|\right)
$$

for any $\varphi \in L^{2}\left(\mathbb{R}^{3}\right)$ with $e(\varphi)<0$. 
Proof The first identity for the norm of the reduced resolvent follows immediately from the definition of the spectral gap $\Lambda(\varphi)$ in (6). For $\psi \in L^{2}\left(\mathbb{R}^{3}\right)$ we have

$$
\left\|(-\Delta+1)^{1 / 2} R_{\varphi}^{1 / 2} \psi\right\|_{2}^{2}=\left\langle\psi, R_{\varphi}^{1(2}(-\Delta+1) R_{\varphi}^{1 / 2} \psi\right\rangle .
$$

It follows from Lemma 2 that there exists $C>0$ such that

$$
\begin{aligned}
\left\|(-\Delta+1)^{1 / 2} R_{\varphi}^{1 / 2} \psi\right\|_{2}^{2} & \leq C\left\langle\psi, R_{\varphi}^{1 / 2}\left(h_{\varphi}+C\|\varphi\|_{2}^{2}\right) R_{\varphi}^{1 / 2} \psi\right\rangle \\
& =C\left\|q_{\psi} \psi\right\|_{2}^{2}+C\left(C\|\varphi\|_{2}^{2}+e(\varphi)\right)\left\|R_{\varphi}^{1 / 2} \psi\right\|_{2}^{2}
\end{aligned}
$$

Since $e(\varphi)<0$ this implies the desired estimate.

\subsection{Perturbative properties of ground states and of the spectral gap}

Since the essential spectrum of $h_{\varphi}$ is $\mathbb{R}_{+}$, the assumption $e(\varphi)<0$ guarantees the existence of a ground state (denoted by $\psi_{\varphi}$ ) and of a spectral gap $\Lambda(\varphi)>0$ of $h_{\varphi}$. In the next two Lemmas we investigate the behavior of $\Lambda(\varphi)$ and $\psi_{\varphi}$ under $L^{2}$-perturbations of $\varphi$.

Lemma 4 Let $\varphi_{0}$ satisfy (8), and let $0<\Lambda<\Lambda\left(\varphi_{0}\right)$. Then, there exists $\delta_{\Lambda}>0$ (depending, besides $\Lambda$, only on the spectrum of $h_{\varphi_{0}}$ and $\left.\left\|\varphi_{0}\right\|_{2}\right)$ such that

$$
\Lambda(\varphi) \geq \Lambda \text { for all } \varphi \in L^{2}\left(\mathbb{R}^{3}\right) \text { with }\left\|\varphi-\varphi_{0}\right\|_{2} \leq \delta_{\Lambda}
$$

Proof By definition of the spectral gap

$$
\Lambda(\varphi)=e_{1}(\varphi)-e(\varphi)
$$

where $e(\varphi)$ denotes the ground state energy of $h_{\varphi}$, and $e_{1}(\varphi)$ its first excited eigenvalue if it exists, or otherwise $e_{1}(\varphi)=0$ (which is the bottom of the essential spectrum). By the min-max principle we can write

$$
e_{1}(\varphi)=\inf _{\substack{A \subset L^{2}\left(\mathbb{R}^{3}\right) \\ \operatorname{dim} A=2}} \sup _{\substack{\psi \in A \\\|\psi\|_{2}=1}}\left\langle\psi, h_{\varphi} \psi\right\rangle
$$

For $\psi \in H^{1}\left(\mathbb{R}^{3}\right)$ with $\|\psi\|_{2}=1$ we find with Lemma 2

$$
\begin{aligned}
\left\langle\psi, h_{\varphi} \psi\right\rangle & =\left\langle\psi, h_{\varphi_{0}} \psi\right\rangle+\left\langle\psi, V_{\varphi-\varphi_{0}} \psi\right\rangle \\
& \leq\left\langle\psi, h_{\varphi_{0}} \psi\right\rangle+C\left\|\varphi-\varphi_{0}\right\|_{2}\|\psi\|_{H^{1}\left(\mathbb{R}^{3}\right)}^{2}
\end{aligned}
$$

Thus, by (18), we have if $\left\|\varphi-\varphi_{0}\right\|_{2} \leq \delta$

$$
\left\langle\psi, h_{\varphi} \psi\right\rangle \leq(1+C \delta)\left\langle\psi, h_{\varphi_{0}} \psi\right\rangle+C \delta\left(\left\|\varphi_{0}\right\|_{2}^{2}+1\right)
$$


and similarly

$$
\left\langle\psi, h_{\varphi} \psi\right\rangle \geq(1-C \delta)\left\langle\psi, h_{\varphi_{0}} \psi\right\rangle-C \delta\left(\left\|\varphi_{0}\right\|_{2}^{2}+1\right)
$$

Since $e\left(\varphi_{0}\right), e\left(\varphi_{1}\right) \leq 0$, we therefore find

$$
\begin{aligned}
\Lambda(\varphi) & \geq \Lambda\left(\varphi_{0}\right)-C \delta\left(e\left(\varphi_{0}\right)+e_{1}\left(\varphi_{0}\right)+2\left(\left\|\varphi_{0}\right\|_{2}^{2}+1\right)\right) \\
& \geq \Lambda\left(\varphi_{0}\right)-2 C \delta\left(\left\|\varphi_{0}\right\|_{2}^{2}+1\right)>\Lambda
\end{aligned}
$$

for sufficiently small $\delta=\delta_{\Lambda}>0$.

Lemma 5 Let $\varphi_{0}$ satisfy (8), and let $\varphi \in L^{2}\left(\mathbb{R}^{3}\right)$ with

$$
\left\|\varphi-\varphi_{0}\right\| \leq \delta_{\varphi_{0}}
$$

for sufficiently small $\delta_{\varphi_{0}}>0$. Then, there exists a unique positive and normalized ground state $\psi_{\varphi}$ of $h_{\varphi}$. Moreover, there exists $C>0$ (independent of $\varphi$ ) such that

$$
\left\|\psi_{\varphi_{0}}-\psi_{\varphi}\right\|_{H^{1}\left(\mathbb{R}^{3}\right)} \leq C\left\|\varphi-\varphi_{0}\right\|_{2} .
$$

Proof We write

$$
\psi_{\varphi}-\psi_{\varphi_{0}}=\int_{0}^{1} d \mu \partial_{\mu} \psi_{\varphi_{\mu}}
$$

with $\varphi_{\mu}=\varphi_{0}+\mu\left(\varphi-\varphi_{0}\right)$. Note that $\psi_{\varphi_{\mu}}$ is well defined for all $\mu \in[0,1]$, since

$$
\left\|\varphi_{\mu}-\varphi_{0}\right\|_{2}=\mu\left\|\varphi-\varphi_{0}\right\|_{2} \leq \mu \delta_{\varphi_{0}} \leq \delta_{\varphi_{0}}
$$

and therefore Lemma 4 guarantees the existence of a spectral gap

$$
\Lambda\left(\varphi_{\mu}\right) \geq \Lambda>0
$$

for sufficiently small $\delta_{\varphi_{0}}$, uniformly in $\mu \in[0,1]$. First-order perturbation theory yields

$$
\partial_{\mu} \psi_{\varphi_{\mu}}=R_{\varphi_{\mu}} V_{\varphi_{0}-\varphi} \psi_{\varphi_{\mu}}
$$

and it follows from Lemma 2 that

$$
\begin{aligned}
\left\|\psi_{\varphi_{0}}-\psi_{\varphi}\right\|_{H^{1}\left(\mathbb{R}^{3}\right)} & \leq \int_{0}^{1} d \mu\left\|R_{\varphi_{\mu}} V_{\varphi-\varphi_{0}} \psi_{\varphi_{\mu}}\right\|_{H^{1}\left(\mathbb{R}^{3}\right)} \\
& \leq C \int_{0}^{1} d \mu\left\|(-\Delta+1)^{1 / 2} R_{\varphi_{\mu}}^{1 / 2}\right\|^{2}\left\|\varphi-\varphi_{0}\right\|_{2} .
\end{aligned}
$$


Lemma 3 shows that

$$
\left\|(-\Delta+1)^{1 / 2} R_{\varphi_{\mu}}\right\| \leq C\left(1+\left\|\varphi_{\mu}\right\|_{2}\left\|R_{\varphi_{\mu}}\right\|\right) .
$$

Since $\left\|\varphi_{\mu}\right\|_{2} \leq\left\|\varphi_{0}\right\|_{2}+\mu\left\|\varphi-\varphi_{0}\right\|_{2} \leq\left\|\varphi_{0}\right\|_{2}+\delta_{\varphi_{0}}$, the bound (41) implies that the right-hand side of (44) is bounded independently of $\mu$. Hence, the desired estimate (38) follows.

\subsection{Pekar functionals}

Recall the definition of the Pekar Functionals $\mathcal{G}, \mathcal{E}$ and $\mathcal{F}$ in (3) and (4), and note that

$$
\mathcal{G}(\psi, \varphi)=\mathcal{E}(\psi)+\left\|\varphi+\sigma_{\psi}\right\|_{2}^{2}
$$

As was shown in [9], $\mathcal{E}$ admits a unique strictly positive and radially symmetric minimizer, which is smooth and will be denoted by $\psi_{\mathrm{P}}$. Moreover, the set of all minimizers of $\mathcal{E}$ coincides with

$$
\Theta\left(\psi_{\mathrm{P}}\right)=\left\{e^{i \theta} \psi_{\mathrm{P}}(\cdot-y) \mid \theta \in[0,2 \pi), y \in \mathbb{R}^{3}\right\}
$$

This clearly implies that the set of minimizers of $\mathcal{F}$ coincides with

$$
\Omega\left(\varphi_{\mathrm{P}}\right)=\left\{\varphi_{\mathrm{P}}(\cdot-y) \mid y \in \mathbb{R}^{3}\right\} \text { with } \varphi_{\mathrm{P}}=-\sigma_{\psi_{\mathrm{P}}}
$$

In the following we prove quadratic lower bounds for the Pekar Functionals $\mathcal{E}$ and $\mathcal{F}$. The key ingredients are the results obtained in [6]. In particular, these results allow to infer, using standard arguments, the following Lemma 6, which provides the quadratic lower bounds for $\mathcal{E}$. (We spell out its proof for completeness in the Appendix; a very similar proof in a slightly different setting is also given in [3]). Based on the bound for $\mathcal{E}$, it is then quite straightforward to obtain the quadratic lower bound for $\mathcal{F}$ in the subsequent Lemma 7.

Lemma 6 (Quadratic Bounds for $\mathcal{E}$ ) There exists a positive constant $\kappa$ such that, for any $L^{2}$-normalized $\psi \in H^{1}\left(\mathbb{R}^{3}\right)$,

$$
\mathcal{E}(\psi)-e_{\mathrm{P}} \geq \kappa \min _{\substack{y \in \mathbb{R}^{3} \\ \theta \in[0,2 \pi)}}\left\|\psi-e^{i \theta} \psi_{\mathrm{P}}(\cdot-y)\right\|_{H^{1}\left(\mathbb{R}^{3}\right)}^{2}=\kappa \operatorname{dist}_{H^{1}\left(\mathbb{R}^{3}\right)}^{2}\left(\psi, \Theta\left(\psi_{\mathrm{P}}\right)\right)
$$

Lemma 7 (Quadratic Bounds for $\mathcal{F}$ ) There exists a positive constant $\tau$ such that, for any $\varphi \in L^{2}\left(\mathbb{R}^{3}\right)$,

$$
\mathcal{F}(\varphi)-e_{\mathrm{P}} \geq \tau \min _{y \in \mathbb{R}^{3}}\left\|\varphi-\varphi_{\mathrm{P}}(\cdot-y)\right\|_{2}^{2}=\tau \operatorname{dist}_{L^{2}\left(\mathbb{R}^{3}\right)}^{2}\left(\varphi, \Omega\left(\varphi_{\mathrm{P}}\right)\right) .
$$


Proof Recalling that

$$
\mathcal{F}(\varphi)=\inf _{\substack{\|\psi\|_{2}=1 \\ \psi \in H^{1}\left(\mathbb{R}^{3}\right)}} \mathcal{G}(\psi, \varphi)
$$

our claim trivially follows by showing that for any $L^{2}$-normalized $\psi \in H^{1}\left(\mathbb{R}^{3}\right)$ and $\varphi \in L^{2}\left(\mathbb{R}^{3}\right)$

$$
\mathcal{G}(\psi, \varphi)-e_{\mathrm{P}} \geq \tau \operatorname{dist}_{L^{2}\left(\mathbb{R}^{3}\right)}^{2}\left(\varphi, \Omega\left(\varphi_{\mathrm{P}}\right)\right)
$$

For any such $\psi$ let $y^{*} \in \mathbb{R}^{3}$ and $\theta^{*} \in[0,2 \pi)$ be such that

$$
\left\|\psi-e^{i \theta^{*}} \psi_{\mathrm{P}}\left(\cdot-y^{*}\right)\right\|_{H^{1}\left(\mathbb{R}^{3}\right)}^{2}=\operatorname{dist}_{H^{1}\left(\mathbb{R}^{3}\right)}^{2}\left(\psi, \Theta\left(\psi_{\mathrm{P}}\right)\right),
$$

and denote $e^{i \theta^{*}} \psi_{\mathrm{P}}\left(\cdot-y^{*}\right)$ by $\psi_{\mathrm{P}}^{*}$. By using the previous Lemma 6 , the fact that $\psi$ and $\psi_{\mathrm{P}}^{*}$ are $L^{2}$-normalized, (19) and completing the square, we obtain for, some positive $\kappa^{*}>0$,

$$
\begin{aligned}
\mathcal{G}(\psi, \varphi)-e_{\mathrm{P}}= & \mathcal{E}(\psi)-e_{\mathrm{P}}+\left\|\varphi+\sigma_{\psi}\right\|_{2}^{2} \geq \kappa\left\|\psi-\psi_{\mathrm{P}}^{*}\right\|_{H^{1}\left(\mathbb{R}^{3}\right)}^{2}+\left\|\varphi+\sigma_{\psi}\right\|_{2}^{2} \\
\geq & \kappa^{*}\left\|\sigma_{\psi}-\sigma_{\psi_{\mathrm{P}}^{*}}\right\|_{2}^{2}+\left\|\varphi+\sigma_{\psi}\right\|_{2}^{2} \\
= & \|\left(1+\kappa^{*}\right)^{1 / 2}\left(\sigma_{\psi_{\mathrm{P}}^{*}}-\sigma_{\psi}\right)-\left(1+\kappa^{*}\right)^{-1 / 2}\left(\varphi+\sigma_{\left.\psi_{\mathrm{P}}^{*}\right) \|_{2}^{2}}\right. \\
& +\frac{\kappa^{*}}{1+\kappa^{*}}\left\|\varphi+\sigma_{\psi_{\mathrm{P}}^{*}}\right\|_{2}^{2} \\
\geq & \frac{\kappa^{*}}{1+\kappa^{*}}\left\|\varphi-\varphi_{\mathrm{P}}\left(\cdot-y^{*}\right)\right\|_{2}^{2} \geq \frac{\kappa^{*}}{1+\kappa^{*}} \operatorname{dist}_{L^{2}\left(\mathbb{R}^{3}\right)}^{2}\left(\varphi, \Omega\left(\varphi_{\mathrm{P}}\right)\right) .
\end{aligned}
$$

This completes the proof of (51), and hence of the Lemma, with $\tau=\kappa^{*} /\left(1+\kappa^{*}\right)$.

Remark 1 The two previous quadratic bounds on $\mathcal{E}$ and $\mathcal{F}$ clearly imply, together with (4), that, for any $L^{2}$-normalized $\psi \in H^{1}\left(\mathbb{R}^{3}\right)$ and any $\varphi \in L^{2}\left(\mathbb{R}^{3}\right)$, having low energy guarantees closeness to the surfaces of minimizers $\Theta\left(\psi_{\mathrm{P}}\right)$ and $\Omega\left(\varphi_{\mathrm{P}}\right)$, i.e.

$$
\begin{aligned}
\mathcal{G}(\psi, \varphi) \leq e_{\mathrm{P}}+\varepsilon & \Rightarrow \mathcal{E}(\psi), \mathcal{F}(\varphi) \leq e_{\mathrm{P}}+\varepsilon \\
& \Rightarrow \operatorname{dist}_{H^{1}}^{2}\left(\psi, \Theta\left(\psi_{\mathrm{P}}\right)\right), \operatorname{dist}_{L^{2}}^{2}\left(\varphi, \Omega\left(\varphi_{\mathrm{P}}\right)\right) \leq C \varepsilon
\end{aligned}
$$

Finally, we exploit the previous estimate to obtain the following Lemma. It states that for couples $(\psi, \varphi)$ which have low energy $\psi$ is close to $\psi_{\varphi}$, the ground state of $h_{\varphi}$, and $\varphi$ is close to $-\sigma_{\psi_{\varphi}}$, in the following sense.

Lemma 8 Let $\varepsilon>0$ be sufficiently small, $\psi \in H^{1}\left(\mathbb{R}^{3}\right)$ be $L^{2}$-normalized, $\varphi \in L^{2}\left(\mathbb{R}^{3}\right)$ and let $(\psi, \varphi)$ be such that

$$
\mathcal{G}(\psi, \varphi) \leq e_{\mathrm{P}}+\varepsilon
$$


Then, $h_{\varphi}$ has a positive ground state $\psi_{\varphi}$, and there exists $C>0$ (independent of $(\psi, \varphi))$ such that

$$
\begin{aligned}
\min _{\theta \in[0,2 \pi)}\left\|\psi-e^{i \theta} \psi_{\varphi}\right\|_{H^{1}\left(\mathbb{R}^{3}\right)}^{2} & \leq C \varepsilon \\
\left\|\varphi+\sigma_{\psi_{\varphi}}\right\|_{2}^{2} & \leq C \varepsilon .
\end{aligned}
$$

Proof Since $\mathcal{F}(\varphi) \leq \mathcal{G}(\psi, \varphi)$ for any $L^{2}$-normalized $\psi \in H^{1}\left(\mathbb{R}^{3}\right)$, Lemma 7 implies that for any $\delta>0$ there exists $\varepsilon_{\delta}>0$ such that $\operatorname{dist}_{L^{2}}\left(\varphi, \Omega\left(\varphi_{\mathrm{P}}\right)\right) \leq \delta$ whenever $\mathcal{G}(\psi, \varphi) \leq e_{\mathrm{P}}+\varepsilon_{\delta}$. Moreover, by Lemma 4 , there exists $\bar{\delta}>0$ such that if $\operatorname{dist}_{L^{2}}\left(\varphi, \Omega\left(\varphi_{\mathrm{P}}\right)\right) \leq \bar{\delta}$ then $\psi_{\varphi}$ exists. We then pick $\varepsilon=\varepsilon_{\bar{\delta}}$ and this guarantees that under the hypothesis of the Lemma $\psi_{\varphi}$ is well defined.

Using Lemmas 6 and 7, the assumption (55) implies that there exist $y_{1}$ and $y_{2}$ such that

$$
\min _{\theta \in[0.2 \pi)}\left\|\psi-e^{i \theta} \psi_{\mathrm{P}}\left(\cdot-y_{1}\right)\right\|_{H^{1}\left(\mathbb{R}^{3}\right)}^{2} \leq C \varepsilon, \quad\left\|\varphi-\varphi_{\mathrm{P}}\left(\cdot-y_{2}\right)\right\|_{2}^{2} \leq C \varepsilon
$$

Moreover, since

$$
e_{\mathrm{P}}+\varepsilon \geq \mathcal{G}(\psi, \varphi)=\mathcal{E}(\psi)+\left\|\varphi+\sigma_{\psi}\right\|_{2}^{2} \geq e_{\mathrm{P}}+\left\|\varphi+\sigma_{\psi}\right\|_{2}^{2},
$$

we also have

$$
\left\|\varphi+\sigma_{\psi}\right\|_{2}^{2} \leq \varepsilon
$$

In combination, the second bound in (58) and (60) imply

$$
\left\|\varphi_{\mathrm{P}}\left(\cdot-y_{2}\right)+\sigma_{\psi}\right\|_{2}^{2} \leq C \varepsilon .
$$

Moreover, with the aid of (19) and the first bound in (58), we obtain

$$
\begin{aligned}
& \left\|\varphi_{\mathrm{P}}\left(\cdot-y_{1}\right)+\sigma_{\psi}\right\|_{2}^{2}=\left\|\sigma_{\psi_{\mathrm{P}}\left(\cdot-y_{1}\right)}-\sigma_{\psi}\right\|_{2}^{2} \\
& \quad \leq C \min _{\theta \in[0,2 \pi)}\left\|\psi-e^{i \theta} \psi_{\mathrm{P}}\left(\cdot-y_{1}\right)\right\|_{H^{1}}^{2} \leq C \varepsilon .
\end{aligned}
$$

By putting the second equation in (58), (61) and (62) together, we can hence conclude that

$$
\begin{aligned}
\left\|\varphi-\varphi_{\mathrm{P}}\left(\cdot-y_{1}\right)\right\|_{2} & \leq\left\|\varphi-\varphi_{\mathrm{P}}\left(\cdot-y_{2}\right)\right\|_{2}+\left\|\varphi_{\mathrm{P}}\left(\cdot-y_{2}\right)+\sigma_{\psi}\right\|_{2}+\left\|\sigma_{\psi}+\varphi_{\mathrm{P}}\left(\cdot-y_{1}\right)\right\|_{2} \\
& \leq C \varepsilon^{1 / 2} .
\end{aligned}
$$

Therefore, using Lemma 5, we obtain

$$
\begin{aligned}
\left\|\psi-e^{i \theta} \psi_{\varphi}\right\|_{H^{1}} & \leq\left\|\psi-e^{i \theta} \psi_{\mathrm{P}}\left(\cdot-y_{1}\right)\right\|_{H^{1}}+\left\|\psi_{\mathrm{P}}\left(\cdot-y_{1}\right)-\psi_{\varphi}\right\|_{H^{1}} \\
& =\left\|\psi-e^{i \theta} \psi_{\mathrm{P}}\left(\cdot-y_{1}\right)\right\|_{H^{1}}+\left\|\psi_{\varphi_{\mathrm{P}}\left(\cdot-y_{1}\right)}-\psi_{\varphi}\right\|_{H^{1}}
\end{aligned}
$$




$$
\leq\left\|\psi-e^{i \theta} \psi_{\mathrm{P}}\left(\cdot-y_{1}\right)\right\|_{H^{1}}+C\left\|\varphi_{\mathrm{P}}\left(\cdot-y_{1}\right)-\varphi\right\|_{2} .
$$

This yields (56) after taking the infimum over $\theta \in[0,2 \pi)$ and using (63) and the first bound in (58). To prove (57), we use (60), (19), the normalization of $\psi$ and $\psi_{\varphi}$ and (56) to obtain

$$
\begin{aligned}
\left\|\varphi+\sigma_{\psi_{\varphi}}\right\|_{2} & \leq\left\|\varphi+\sigma_{\psi}\right\|_{2}+\left\|\sigma_{\psi}-\sigma_{\psi_{\varphi}}\right\|_{2} \\
& \leq \varepsilon^{1 / 2}+C \min _{\theta \in[0,2 \pi)}\left\|\psi-e^{i \theta} \psi_{\varphi}\right\|_{H^{1}} \leq C \varepsilon^{1 / 2} .
\end{aligned}
$$

\section{Proof of the main results}

The conservation of $\mathcal{G}$ along solutions of the Landau-Pekar equations allows to apply the tools developed in Sect. 2 to get results valid for all times. This will in particular allow us to prove the results stated in Sect. 1. When combined with energy conservation, Remark 1 shows that we can estimate the distance to the sets of Pekar minimizers of solutions of the Landau-Pekar equations only in terms of the energy of their initial data. Since $\Omega\left(\varphi_{\mathrm{P}}\right)$ contains only real-valued functions this yields bounds on the $L^{2}$ norm of the imaginary part of $\varphi_{t}$. That is, there exists a $C>0$ such that if $\left(\psi_{t}, \varphi_{t}\right)$ solves the Landau-Pekar equations (1) with initial data $\left(\psi_{0}, \varphi_{0}\right)$, then

$$
\begin{aligned}
& \min _{\substack{y \in \mathbb{R}^{3} \\
\theta \in[0,2 \pi)}}\left\|\psi_{t}-e^{i \theta} \psi_{\mathrm{P}}(\cdot-y)\right\|_{H^{1}\left(\mathbb{R}^{3}\right)}^{2} \leq C\left(\mathcal{G}\left(\psi_{0}, \varphi_{0}\right)-e_{\mathrm{P}}\right), \\
& \left\|\operatorname{Im} \varphi_{t}\right\|_{2}^{2} \leq C\left(\mathcal{G}\left(\psi_{0}, \varphi_{0}\right)-e_{\mathrm{P}}\right), \\
& \min _{y \in \mathbb{R}^{3}}\left\|\operatorname{Re} \varphi_{t}-\varphi_{\mathrm{P}}(\cdot-y)\right\|_{2}^{2} \leq C\left(\mathcal{G}\left(\psi_{0}, \varphi_{0}\right)-e_{\mathrm{P}}\right)
\end{aligned}
$$

for all $t \in \mathbb{R}$ and $\alpha>0$. It is then straightforward to obtain a proof of Theorem 1 .

Proof of Theorem 1 Let $0<\Lambda<\Lambda\left(\varphi_{\mathrm{P}}\right)$ and let $\left(\psi_{t}, \varphi_{t}\right)$ denote the solution to the Landau-Pekar equations with initial data $\left(\psi_{0}, \varphi_{0}\right)$ satisfying $\mathcal{G}\left(\psi_{0}, \varphi_{0}\right) \leq e_{\mathrm{P}}+\varepsilon_{\Lambda}$. From (66) we deduce that for any $t \in \mathbb{R}$ there exists $y_{t} \in \mathbb{R}^{3}$ such that

$$
\left\|\varphi_{t}-\varphi_{\mathrm{P}}\left(\cdot-y_{t}\right)\right\|_{2}^{2} \leq C \varepsilon_{\Lambda}
$$

for some $C>0$. Since the spectrum of $h_{\varphi_{\mathrm{P}}(\cdot-y)}$ and $\left\|\varphi_{\mathrm{P}}(\cdot-y)\right\|_{2}$ are independent of $y \in \mathbb{R}^{3}$, Theorem 1 now follows immediately from Lemma 4 by taking $\varepsilon_{\Lambda}=C^{-1} \delta_{\Lambda}^{2}$, where $\delta_{\Lambda}$ is the same as in Lemma 4 .

Conservation of energy also allows to extend the validity of Lemma 8 for all times. If $\left(\psi_{t}, \varphi_{t}\right)$ solves (1) with initial data $\left(\psi_{0}, \varphi_{0}\right)$ satisfying $\mathcal{G}\left(\psi_{0}, \varphi_{0}\right) \leq e_{\mathrm{P}}+\varepsilon$ for a sufficiently small $\varepsilon$, then $\psi_{\varphi_{t}}$ is well defined for all times and

$$
\min _{\theta \in[0,2 \pi)}\left\|\psi_{t}-e^{i \theta} \psi_{\varphi_{t}}\right\|_{H^{1}\left(\mathbb{R}^{3}\right)}^{2} \leq C \varepsilon, \quad\left\|\varphi_{t}+\sigma_{\psi_{\varphi_{t}}}\right\|_{2}^{2} \leq C \varepsilon .
$$


Moreover, Theorem 1 implies that for all times $\Lambda\left(\varphi_{t}\right) \geq \Lambda$ for a suitable $\Lambda>0$. It thus follows from Lemmas 1 and 3 that for some $C>0$

$$
\left\|R_{\varphi_{t}}\right\| \leq C \text { and }\left\|(-\Delta+1)^{1 / 2} R_{\varphi_{t}}^{1 / 2}\right\| \leq C \text { for all } t \in \mathbb{R}
$$

whereas above $R_{\varphi_{t}}=q_{t}\left(h_{\varphi_{t}}-e\left(\varphi_{t}\right)\right)^{-1} q_{t}$ and $q_{t}=1-p_{t}=1-\left|\psi_{\varphi_{t}}\right\rangle\left\langle\psi_{\varphi_{t}}\right|$.

With these preparations, we are now ready to prove Corollary 1.

Proof of Corollary 1 The proof follows closely the ideas of the proof of [8, Theorem II.1], hence we allow ourselves to be a bit sketchy at some points and refer to [8] for more details. It follows from the Landau-Pekar equations (1) that

$$
\alpha^{2} \partial_{t} V_{\varphi_{t}}=V_{\operatorname{Im} \varphi_{t}}, \quad \alpha^{2} \partial_{t} V_{\operatorname{Im} \varphi_{t}}=-V_{\operatorname{Re} \varphi_{t}+\sigma_{\psi_{t}}}
$$

Lemmas 1-3 imply, together with (66), that there exists $C>0$ such that

$$
\left\|R_{\varphi_{t}} V_{\operatorname{Im} \varphi_{t}}\right\|^{2} \leq C \varepsilon \text { for all } t \in \mathbb{R} .
$$

In the same way, by the triangle inequality, Lemma 2 and (68), there exists $C>0$ such that

$$
\begin{aligned}
\left\|R_{\varphi_{t}} V_{\operatorname{Re} \varphi_{t}+\sigma_{\psi_{t}}}\right\|^{2} & \leq C \min _{\theta \in(0,2 \pi]}\left\|\psi_{t}-e^{i \theta} \psi_{\varphi_{t}}\right\|_{H^{1}\left(\mathbb{R}^{3}\right)}^{2}+C\left\|\operatorname{Re} \varphi_{t}+\sigma_{\psi_{\varphi_{t}}}\right\|_{2}^{2} \\
& \leq C \varepsilon \text { for all } t \in \mathbb{R} .
\end{aligned}
$$

Moreover, it follows from

$$
\alpha^{2} \partial_{t} \psi_{\varphi_{t}}=-R_{\varphi_{t}} V_{\operatorname{Im} \varphi_{t}} \psi_{\varphi_{t}}
$$

that

$$
\alpha^{2} \partial_{t} R_{\varphi_{t}}=p_{t} V_{\operatorname{Im} \varphi_{t}} R_{\varphi_{t}}^{2}+R_{\varphi_{t}}^{2} V_{\operatorname{Im} \varphi_{t}} p_{t}-R_{\varphi_{t}}\left(V_{\operatorname{Im} \varphi_{t}}-\left\langle\psi_{\varphi_{t}}, V_{\operatorname{Im} \varphi_{t}} \psi_{\varphi_{t}}\right\rangle\right) R_{\varphi_{t}}
$$

(see [8, Lemma IV.2]) and by the same arguments as above that

$$
\left\|(-\Delta+1)^{1 / 2} \partial_{t} R_{\varphi_{t}}(-\Delta+1)^{1 / 2}\right\| \leq C \varepsilon^{1 / 2} \alpha^{-2} \text { for all } t \in \mathbb{R} .
$$

Recall the definitions of $\widetilde{\psi}_{t}$ and $v$ in (11). The same computations as in [8, Eqs. (58)(65)], using

$$
q_{t} e^{i \int_{0}^{t} d s e\left(\varphi_{s}\right)} \psi_{t}=i R_{\varphi_{t}} \partial_{t} e^{i \int_{0}^{t} d s e\left(\varphi_{s}\right)} \psi_{t}
$$

and integration by parts, lead to

$$
\left\|\widetilde{\psi}_{t}-\psi_{\varphi_{t}}\right\|_{2}^{2}=2 \alpha^{-2} \operatorname{Im}\left\langle\widetilde{\psi}_{t}, R_{\varphi_{t}}^{2} V_{\operatorname{Im} \varphi_{t}} \psi_{\varphi_{t}}\right\rangle
$$




$$
\begin{aligned}
& +2 \alpha^{-2} \int_{0}^{t} d s v(s) \operatorname{Re}\left\langle\widetilde{\psi}_{s}, R_{\varphi_{s}}^{2} V_{\operatorname{Im} \varphi_{s}} \psi_{\varphi_{s}}\right\rangle \\
& +2 \alpha^{-4} \int_{0}^{t} d s \operatorname{Im}\left\langle\widetilde{\psi}_{s}, R_{\varphi_{s}}\left(R_{\varphi_{s}} V_{\operatorname{Im} \varphi_{s}}\right)^{2} \psi_{\varphi_{s}}\right\rangle \\
& +2 \alpha^{-4} \int_{0}^{t} d s \operatorname{Im}\left\langle\widetilde{\psi}_{s}, R_{\varphi_{s}}^{2} V_{\operatorname{Re} \varphi_{s}+\sigma_{\psi_{s}}} \psi_{\varphi_{s}}\right\rangle \\
& -2 \alpha^{-2} \int_{0}^{t} d s\left(\operatorname{Im}\left\langle\widetilde{\psi}_{s},\left(\partial_{s} R_{\varphi_{s}}^{2}\right) V_{\operatorname{Im} \varphi_{s}} \psi_{\varphi_{s}}\right\rangle+\alpha^{2} v(s) \operatorname{Im}\left\langle\widetilde{\psi}_{s}, \psi_{\varphi_{s}}\right\rangle\right)
\end{aligned}
$$

The difference to the calculations in [8] are the additional terms (77b) and the second term in $(77 \mathrm{e})$ resulting from the phase $v$. While (77b) is, as we show below, only a subleading error term, the phase in $(77 \mathrm{e})$ leads to a crucial cancellation. This cancellation allows to integrate by parts once more, and finally results in the improved estimate in Corollary 1.

We shall now estimate the various terms in (77). Since $\left\|q_{t} \widetilde{\psi}_{t}\right\|_{2} \leq\left\|\widetilde{\psi}_{t}-\psi_{\varphi_{t}}\right\|_{2}$, we find for the first term using (69) and (71)

$$
|(77 a)| \leq C \alpha^{-2} \varepsilon^{1 / 2}\left\|\widetilde{\psi}_{t}-\psi_{\varphi_{t}}\right\|_{2} \leq \delta\left\|\widetilde{\psi}_{t}-\psi_{\varphi_{t}}\right\|_{2}^{2}+C \delta^{-1} \alpha^{-4} \varepsilon
$$

for arbitrary $\delta>0$. Moreover, we have $|v(s)| \leq C \alpha^{-4} \varepsilon$ for all $s \in \mathbb{R}$, and find for the second term

$$
|(77 b)| \leq C \alpha^{-6} \varepsilon^{3 / 2} \int_{0}^{t} d s\left\|\widetilde{\psi}_{s}-\psi_{\varphi_{s}}\right\|_{2} .
$$

For the third term, we integrate by parts using (76) once more, with the result that

$$
\begin{aligned}
(77 c)= & -2 \alpha^{-4} \operatorname{Re}\left\langle\widetilde{\psi}_{t}, R_{\varphi_{t}}^{2}\left(R_{\varphi_{t}} V_{\operatorname{Im} \varphi_{t}}\right)^{2} \psi_{\varphi_{t}}\right\rangle \\
& +2 \alpha^{-4} \int_{0}^{t} d s v(s) \operatorname{Im}\left\langle\widetilde{\psi}_{s}, R_{\varphi_{s}}^{2}\left(R_{\varphi_{s}} V_{\operatorname{Im} \varphi_{s}}\right)^{2} \psi_{\varphi_{s}}\right\rangle \\
& +2 \alpha^{-4} \int_{0}^{t} d s \operatorname{Re}\left\langle\widetilde{\psi}_{s}, \partial_{s}\left(R_{\varphi_{s}}^{2}\left(R_{\varphi_{s}} V_{\operatorname{Im} \varphi_{s}}\right)^{2} \psi_{\varphi_{s}}\right)\right\rangle
\end{aligned}
$$

The first two terms can be bounded in the same way as (77a) and (77b). For the third term, note that the r.h.s. of the inner product depends on time $s$ through $\varphi_{s}$ only, hence its time derivative leads to another factor of $\alpha^{-2}$. With (70), (73) and (74) we compute its time derivative. From the time derivative of the reduced resolvent in (74), we obtain one term for which the projection $p_{s}$ hits $\widetilde{\psi}_{s}$ on the l.h.s. of the inner product, in which case we can only bound $\left\|p_{s} \widetilde{\psi}_{s}\right\|_{2} \leq 1$. For the remaining terms, we use $\left\|q_{s} \widetilde{\psi}_{s}\right\|_{2} \leq\left\|\widetilde{\psi}_{s}-\psi_{\varphi_{s}}\right\|_{2}$ instead. With the same arguments as above and (72), we obtain 


$$
\begin{aligned}
|(77 c)| \leq & \delta\left\|\widetilde{\psi}_{t}-\psi_{\varphi_{t}}\right\|_{2}^{2}+C \delta^{-1} \alpha^{-8} \varepsilon^{2} \\
& +C \alpha^{-6} \varepsilon \int_{0}^{t} d s\left\|\widetilde{\psi}_{s}-\psi_{\varphi_{s}}\right\|_{2}+C \alpha^{-6} \varepsilon^{3 / 2}|t|
\end{aligned}
$$

for any $\delta>0$. For the fourth term (77d), we first split

$$
(77 d)=2 \alpha^{-4} \int_{0}^{t} d s\left(\operatorname{Im}\left\langle\widetilde{\psi}_{s}, R_{\varphi_{s}}^{2} V_{\sigma_{\psi_{s}}-\sigma_{\psi_{s}}} \psi_{\varphi_{s}}\right\rangle+\operatorname{Im}\left\langle\widetilde{\psi}_{s}, R_{\varphi_{s}}^{2} V_{\operatorname{Re} \varphi_{s}+\sigma_{\psi_{\varphi_{s}}}} \psi_{\varphi_{s}}\right\rangle\right)
$$

Lemmas 1-3 and (69) imply that we can bound $\left\|R_{\varphi_{s}}^{2} V_{\sigma_{\psi_{s}}-\sigma_{\psi_{\varphi_{s}}}}\right\| \leq C\left\|\widetilde{\psi}_{s}-\psi_{\varphi_{s}}\right\|_{2}$ in the first term. For the second term, we observe that the r.h.s. of the inner product depends on $s$ again only through $\varphi_{s}$, whose time derivative is of order $\alpha^{-2}$. We thus again use (76) and integration by parts, and proceed as above. For the calculation, we need to bound the time derivative of $\sigma_{\psi_{\varphi_{s}}}$, which can be done with the aid [7, Lemma II.4], with the result that $\left\|\partial_{s} \sigma_{\psi_{\varphi_{s}}}\right\|_{2} \leq C \varepsilon^{1 / 2} \alpha^{-2}$. Altogether, this shows that

$$
\begin{aligned}
|(77 d)| \leq & C \alpha^{-4} \int_{0}^{t} d s\left\|\widetilde{\psi}_{s}-\psi_{\varphi_{s}}\right\|_{2}^{2}+\delta\left\|\widetilde{\psi}_{t}-\psi_{\varphi_{t}}\right\|_{2}^{2}+C \delta^{-1} \alpha^{-8} \varepsilon \\
& +C \alpha^{-6} \varepsilon^{1 / 2} \int_{0}^{t} d s\left\|\widetilde{\psi}_{s}-\psi_{\varphi_{s}}\right\|_{2}+C \alpha^{-6} \varepsilon|t|
\end{aligned}
$$

for any $\delta>0$. For the last term, we compute using (74)

$$
\begin{aligned}
(77 e)= & -6 \alpha^{-4} \int_{0}^{t} d s \operatorname{Im}\left\langle\widetilde{\psi}_{s}, R_{\varphi_{s}}^{3} V_{\operatorname{Im} \varphi_{s}} p_{s} V_{\operatorname{Im} \varphi_{s}} \psi_{\varphi_{s}}\right\rangle \\
& +2 \alpha^{-4} \int_{0}^{t} d s \operatorname{Im}\left\langle\widetilde{\psi}_{s},\left(R_{\varphi_{s}}^{2} V_{\operatorname{Im} \varphi_{s}} R_{\varphi_{s}}+R_{\varphi_{s}} V_{\operatorname{Im} \varphi_{s}} R_{\varphi_{s}}^{2}\right) V_{\operatorname{Im} \varphi_{s}} \psi_{\varphi_{s}}\right\rangle .
\end{aligned}
$$

Note that the phase $\nu(s)$ cancels the contribution of $\partial_{s} R_{\varphi_{s}}$ projecting onto $\psi_{\varphi_{s}}$ (the first term of (74)). This cancellation is important, since the integration by parts argument using (76) would not be applicable to this term. It can be applied to all the terms in (84), however, proceeding as above, with the result that

$$
\begin{aligned}
|(77 e)| \leq \delta & \left\|\widetilde{\psi}_{t}-\psi_{\varphi_{t}}\right\|_{2}^{2}+C \delta^{-1} \alpha^{-8} \varepsilon^{2} \\
& +C \alpha^{-6} \varepsilon \int_{0}^{t} d s\left\|\widetilde{\psi}_{s}-\psi_{\varphi_{s}}\right\|_{2}+C \alpha^{-6} \varepsilon^{3 / 2}|t|
\end{aligned}
$$

for any $\delta>0$.

Collecting the bounds in (78), (79), (81), (83) and (85), Eq. (77) shows that 


$$
\begin{aligned}
\left\|\widetilde{\psi}_{t}-\psi_{\varphi_{t}}\right\|_{2}^{2} \leq & C \alpha^{-4} \varepsilon+C \alpha^{-6} \varepsilon^{1 / 2} \int_{0}^{t} d s\left\|\widetilde{\psi}_{s}-\psi_{\varphi_{s}}\right\|_{2} \\
& +C \alpha^{-4} \int_{0}^{t} d s\left\|\widetilde{\psi}_{s}-\psi_{\varphi_{s}}\right\|_{2}^{2}+C \alpha^{-6} \varepsilon|t| \\
\leq & C \alpha^{-4} \varepsilon+C \alpha^{-4} \int_{0}^{t} d s\left\|\widetilde{\psi}_{s}-\psi_{\varphi_{s}}\right\|_{2}^{2}+C \alpha^{-6} \varepsilon|t|
\end{aligned}
$$

for $\alpha \gtrsim 1$ and $\varepsilon \lesssim 1$. A Gronwall type argument finally yields the desired bound (12).

Acknowledgements Funding from the European Union's Horizon 2020 research and innovation programme under the ERC Grant Agreement No 694227 (D.F. and R.S.) and under the Marie Skłodowska-Curie Grant Agreement No. 754411 (S.R.) is gratefully acknowledged.

Funding Open Access funding provided by Institute of Science and Technology (IST Austria)

Open Access This article is licensed under a Creative Commons Attribution 4.0 International License, which permits use, sharing, adaptation, distribution and reproduction in any medium or format, as long as you give appropriate credit to the original author(s) and the source, provide a link to the Creative Commons licence, and indicate if changes were made. The images or other third party material in this article are included in the article's Creative Commons licence, unless indicated otherwise in a credit line to the material. If material is not included in the article's Creative Commons licence and your intended use is not permitted by statutory regulation or exceeds the permitted use, you will need to obtain permission directly from the copyright holder. To view a copy of this licence, visit http://creativecommons.org/licenses/by/4.0/.

\section{Appendix: Proof of Lemma 6}

In this appendix we give the proof of Lemma 6. As already mentioned, the result follows from the work in [6] by standard arguments. We follow closely the proof given in [3] of a corresponding result in the slightly different setting of a confined polaron.

Proof of Lemma 6 Step 1: For any $L^{2}$-normalized $\psi \in H^{1}\left(\mathbb{R}^{3}\right)$, there exists $\bar{\theta} \in$ $[0,2 \pi)$ and $\bar{y} \in \mathbb{R}^{3}$ such that

$$
\left\|e^{i \bar{\theta}} \psi(\cdot-\bar{y})-\psi_{\mathrm{P}}\right\|_{2}=\min _{y, \theta}\left\|e^{i \theta} \psi(\cdot-y)-\psi_{\mathrm{P}}\right\|_{2}
$$

By invariance of $\mathcal{E}$ under translations and changes of phase, it is then sufficient to show that for any $L^{2}$-normalized $\psi$ such that

$$
\left\|\psi-\psi_{\mathrm{P}}\right\|_{2}=\min _{y, \theta}\left\|\psi-e^{i \theta} \psi_{\mathrm{P}}(\cdot-y)\right\|_{2},
$$

the inequality

$$
\mathcal{E}(\psi)-e_{\mathrm{P}} \geq \kappa\left\|\psi-\psi_{\mathrm{P}}\right\|_{H^{1}\left(\mathbb{R}^{3}\right)}^{2}
$$


holds (for some $\kappa>0$ independent of $\psi$ ). In fact, this is stronger than the desired bound (48). We henceforth only work with $L^{2}$-normalized $\psi$ satisfying (88), and denote $\delta=\psi-\psi_{\mathrm{P}}$. Observe that any $\psi$ satisfying (88) also satisfies

$$
\left\langle\psi \mid \psi_{\mathrm{P}}\right\rangle \geq 0, \quad\left\langle\psi \mid \partial_{i} \psi_{\mathrm{P}}\right\rangle=0 \text { for } i=1,2,3 \text {. }
$$

Step 2: We first prove the quadratic lower bound (89) locally around $\psi_{\mathrm{P}}$ for any $L^{2}$-normalized $\psi$ satisfying (88). By straightforward computations, using that

$$
\|\delta\|_{2}^{2}=2-2\left\langle\psi_{\mathrm{P}} \mid \psi\right\rangle=-2\left\langle\psi_{\mathrm{P}} \mid \delta\right\rangle
$$

since both $\psi_{\mathrm{P}}$ and $\psi$ are $L^{2}$-normalized, we obtain

$$
\mathcal{E}(\psi)-e_{\mathrm{P}}=\operatorname{Hess}_{\psi_{\mathrm{P}}}(\delta)+O\left(\|\delta\|_{H^{1}\left(\mathbb{R}^{3}\right)}^{3}\right),
$$

with

$$
\begin{aligned}
\operatorname{Hess}_{\psi_{\mathrm{P}}}(\delta) & =\left\langle\operatorname{Im} \delta\left|Q L_{-} Q\right| \operatorname{Im} \delta\right\rangle+\left\langle\operatorname{Re} \delta\left|Q L_{+} Q\right| \operatorname{Re} \delta\right\rangle, \\
Q & =1-\left|\psi_{\mathrm{P}}\right\rangle\left\langle\psi_{\mathrm{P}}\right|, \\
L_{-} & =h_{\varphi_{\mathrm{P}}}-e\left(\varphi_{\mathrm{P}}\right), \\
L_{+} & =L_{-}-4 X \\
X & =(2 \pi)^{3} \psi_{\mathrm{P}}(-\Delta)^{-1} \psi_{\mathrm{P}},
\end{aligned}
$$

where in the last formula for $X, \psi_{\mathrm{P}}$ has to be understood as a multiplication operator.

The Euler-Lagrange equation for the minimization of $\mathcal{E}$ reads $L_{-} \psi_{\mathrm{P}}=0$, and since $L_{-}$is a Schrödinger operator and $\psi_{\mathrm{P}}$ is strictly positive, $L_{-}$has 0 as its lowest eigenvalue, and a gap above. Therefore, we have

$$
Q L_{-} Q \geq \kappa_{1} Q
$$

for some $\kappa_{1}>0$. Moreover, it was shown in [6] that the kernel of $L_{+}$coincides with $\operatorname{span}_{i=1,2,3}\left\{\partial_{i} \psi_{\mathrm{P}}\right\}$ and from this we can infer the existence of a $\kappa_{2}>0$ such that

$$
Q L_{+} Q \geq \kappa_{2} Q^{\prime} \quad \text { with } Q^{\prime}=Q-\sum_{i=1}^{3}\left\|\partial_{i} \psi_{\mathrm{P}}\right\|_{2}^{-2}\left|\partial_{i} \psi_{\mathrm{P}}\right\rangle\left\langle\partial_{i} \psi_{\mathrm{P}}\right|
$$

Recall that $Q^{\prime} \delta=Q \delta$ by assumption on $\psi$ and orthogonality of $\psi_{\mathrm{P}}$ to its partial derivatives. With $\kappa^{\prime}=\min \left\{\kappa_{1}, \kappa_{2}\right\}$ we thus have

$$
\operatorname{Hess}_{\psi_{\mathrm{P}}}(\delta) \geq \kappa_{1}\|Q \operatorname{Im} \delta\|_{2}^{2}+\kappa_{2}\left\|Q^{\prime} \operatorname{Re} \delta\right\|_{2}^{2} \geq \kappa^{\prime}\|Q \delta\|_{2}^{2}
$$

Using again (91) we see that

$$
\|Q \delta\|_{2}^{2}=\|\delta\|_{2}^{2}-\left\langle\psi_{\mathrm{P}} \mid \delta\right\rangle^{2}=\|\delta\|_{2}^{2}\left(1-\frac{1}{4}\|\delta\|_{2}^{2}\right) \geq \frac{1}{2}\|\delta\|_{2}^{2},
$$


which finally implies that

$$
\operatorname{Hess}_{\psi_{\mathrm{P}}}(\delta) \geq \frac{\kappa^{\prime}}{2}\|\delta\|_{2}^{2}
$$

We now want to improve this bound to include the full $H^{1}$-norm of $\delta$. Using the regularity of $\psi_{\mathrm{P}}$ it is rather straightforward to show that

$$
\begin{aligned}
L_{-}= & Q L_{-} Q \geq-\Delta-C, \\
& Q L_{+} Q \geq-\Delta-C
\end{aligned}
$$

which implies that

$$
\operatorname{Hess}_{\psi_{\mathrm{P}}}(\delta) \geq\|\delta\|_{H^{1}}^{2}-C\|\delta\|_{2}^{2}
$$

By interpolating between (98) and (100), we finally obtain

$$
\operatorname{Hess}_{\psi_{\mathrm{P}}}(\delta) \geq \frac{\kappa^{\prime}}{\kappa^{\prime}+2 C}\|\delta\|_{H^{1}}^{2}=\kappa^{\prime \prime}\|\delta\|_{H^{1}}^{2}
$$

In combination with (92), we conclude that

$$
\mathcal{E}(\psi)-e_{\mathrm{P}} \geq \kappa^{\prime \prime}\|\delta\|_{H^{1}}^{2}-C\|\delta\|_{H^{1}}^{3}
$$

for any $L^{2}$-normalized $\psi$ satisfying (88), which shows that (89) holds for $\|\delta\|_{H^{1}}$ sufficiently small.

Step 3: We now extend the previous local bound to show that (89) holds globally. Suppose by contradiction that there does not exist a universal $\kappa$ such that (89) holds. Then, there exists a sequence $\psi_{n}$ of $L^{2}$-normalized functions satisfying (88) such that

$$
\mathcal{E}\left(\psi_{n}\right) \leq e_{\mathrm{P}}+\frac{1}{n}\left\|\psi_{n}-\psi_{\mathrm{P}}\right\|_{H^{1}}^{2} \leq \frac{2}{n}\left\|\psi_{n}\right\|_{H^{1}}^{2}+C
$$

One readily checks that

$$
\mathcal{E}\left(\psi_{n}\right) \geq \frac{1}{2}\left\|\psi_{n}\right\|_{H^{1}}^{2}-C,
$$

hence $\psi_{n}$ must be bounded in $H^{1}\left(\mathbb{R}^{3}\right)$. Again using (103), we conclude that $\psi_{n}$ must be a minimizing sequence for $\mathcal{E}$. It was proven in [9] that any minimizing sequence converges in $H^{1}\left(\mathbb{R}^{3}\right)$ to a minimizer of $\mathcal{E}$, i.e., an element of $\Theta\left(\psi_{\mathrm{P}}\right)$ in (46), and since $\psi_{n}$ satisfies (88) this implies that $\psi_{n} \stackrel{H^{1}}{\rightarrow} \psi_{\mathrm{P}}$. This yields a contradiction, since we already know by (102) that locally the bound (89) holds. 


\section{References}

1. Frank, R.L., Gang, Z.: Derivation of an effective evolution equation for a strongly coupled polaron. Anal. PDE 10(2), 379-422 (2017)

2. Frank, R.L., Gang, Z.: A non-linear adiabatic theorem for the one-dimensional Landau- Pekar equations. J. Funct. Anal. 279, 7 (2020)

3. Frank, R. L., Seiringer, R.: Quantum corrections to the Pekar asymptotics of a strongly coupled polaron. Commun. Pure Appl. Math. 74, 544-588 (2021)

4. Fröhlich, H.: Theory of electrical breakdown in ionic crystals. Proc. R. Soc. Lond. A 160(901), 230-241 (1937)

5. Landau, L.D., Pekar, S.I.: Effective mass of a polaron. Zh. Eksp. Teor. Fiz. 18(5), 419-423 (1948)

6. Lenzmann, E.: Uniqueness of ground states for pseudorelativistic Hartree equations. Anal. PDE 2, 1-27 (2009)

7. Leopold, N., Mitrouskas, D., Rademacher, S., Schlein, B., Seiringer, R.: Landau-Pekar equations and quantum fluctuations for the dynamics of a strongly coupled polaron. Preprint: arXiv:2005.02098

8. Leopold, N., Rademacher, S., Schlein, B., Seiringer, R.: The Landau-Pekar equations: Adiabatic theorem and accuracy. Preprint: arXiv:1904.12532, Analysis \& PDE (in press)

9. Lieb, E.H.: Existence and uniqueness of the minimizing solution of Choquard's nonlinear equation. Stud. Appl. Math. 57, 93 (1977)

10. Mitrouskas, D.: A note on the Fröhlich dynamics in the strong coupling limit, preprint arXiv:2003.11448

Publisher's Note Springer Nature remains neutral with regard to jurisdictional claims in published maps and institutional affiliations. 